\title{
Ratings of Physicians Relying on Experts Versus Physicians Relying on Decision Aids
}

\author{
C. Adam Probst *, Victoria A. Shaffer, Charles Lambdin, Hal R. Arkes, \& Mitchel A. \\ Medow \\ Department of Psychology, Fairmount College of Liberal Arts and Sciences
}

\begin{abstract}
Errors in the field of medicine are costly, both in terms of monetary expenses and the cost of lives. Computerbased decision aids have been shown to decrease physician error. These decision aids decreased many types of errors, reduced hospital admissions and costs and fostered the implementation of evidence-based medicine. However, patients give lower ratings to physicians who use such aids on many variables (diagnostic ability, professionalism, overall satisfaction with exam).

The current research attempts to ascertain whether patients are uncomfortable with the use of a computer in a humancentered field or the consultation of an outside source by a physician. Participants were asked to evaluate physicians in one of three scenarios: the physician makes an unaided diagnosis, the physician consults a human expert for a second opinion, or the physician uses a computer-based diagnostic aid to diagnose a patient.

Two planned Man-Witney U tests showed that physicians who used the computer-based aid were given lower ratings on diagnostic ability, professionalism, and overall satisfaction compared to physicians making unaided diagnoses. Ratings of physician making unaided diagnosis were not significantly different from that of the physician who consulted human experts. We conclude that it is not the consultation of an outside source that patients find objectionable. However, the use of a computer in a human-centered field such as medicine is responsible for the derogated ratings of physicians.
\end{abstract}

\section{Introduction}

Medical errors are responsible for the loss of 98,000 lives annually and are considered to be the 8th leading cause of death [1]. The monetary expense of these errors is also noteworthy [2]. One type of medical error, adverse drug effects, is estimated to cost hospitals $\$ 2$ billion annually [3]. Malpractice costs and the costs to patients and families are immeasurable [3]. Sadly, medical errors are much more common than many realize. It has been estimated that close to 25 percent of outpatients are subject to medical errors and, on average, a patient in an intensive care unit must endure one healthcare error per day [4].

Computerized diagnostic aids have been shown to reduce physician error. Physician order entry systems have reduced medication errors by 55\% [3]. Management programs have reduced adverse drug reactions by over $70 \%$ [5]. And alert systems have reduced the time between reduction or cessation of medication by over 20 hours [6].

However, these aids are not well received by patients or physicians. Physicians believe that to use such aids is to employ "cookbook" medicine, and undermine years of schooling and training. Patients give lower ratings of diagnostic ability, professionalism, and overall satisfaction to physicians using a computer-based aid during diagnosis [7].

The goal of this research is to identify whether these lower ratings are due to the use of a computer in a human-centered field such as medicine, or the reliance on an external source. Participants were asked to evaluate physicians in one of three scenarios: the physician makes an unaided diagnosis, the physician uses a diagnostic aid, or the physician consults a human expert.

\section{Experiment, Results, Discussion, and Significance}

Students from both Wichita State University $(\mathrm{N}=196)$ and Ohio State University $(\mathrm{N}=238)$ volunteered to participate. Participants were awarded course/extra credit for completion of the experiment. Participants completed a computerized questionnaire that was administered using Media Lab software. 
Participants were randomly assigned to one of three conditions $(\mathrm{N}$ control $=145, \mathrm{~N}$ expert=141, $\mathrm{N}$ aid=148). They were asked to read a scenario in which they were taken to a physician because of an ankle injury received while on a skiing trip. In the control condition, the physician made an unaided diagnosis. In the expert condition, the physician consulted another human expert for a second opinion. And in the aid condition, the physician consulted a computer based diagnostic aid. Participants were asked to take the role of the patient and rate the physician on the following dimensions ( $1=$ less positive to $7=$ most positive); diagnostic ability, professionalism, overall satisfaction, thoroughness of exam, and length of wait.

There were no significant differences due to gender or ethnicity. Heterogeneity of variance was observed among several of the dependent variables. Therefore, two planned Mann-Whitney $U$ tests were used to compare the control group with the aid group and the control group with the expert group.

The physician making the unaided diagnosis was rated more positively than the physician using the computer based aid on three variables (diagnostic ability: $z=-1.75, p<.05$, overall satisfaction: $z=-1.71, p<.05$, and professionalism: $z=-2.77, p<.05$ ). The physician who consulted a human expert had ratings that were not significantly different than the physician making the unaided diagnosis.

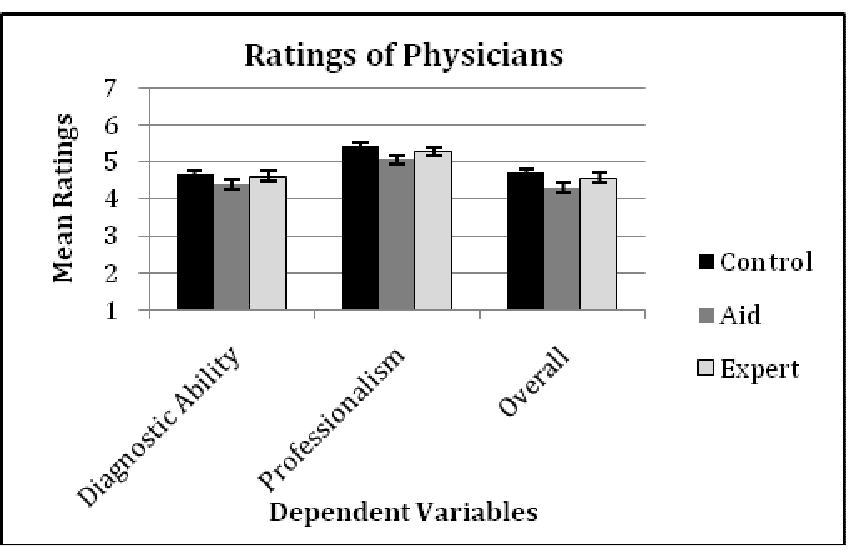

\section{Conclusions}

This study replicated the previous finding that physicians who use computer based diagnostic aids are rated less favorably across several variables than physicians who make an unaided diagnosis [7]. This research demonstrates that it is not the consultation of an outside source that patients find objectionable. Rather, the use of a computer in a human-centered field is responsible for the derogated ratings of physicians.

[1] Brennan, T.A., Leape, L.L., \& Laird, N.M. (1991). Incidence of adverse events and negligence in hospitalized patients: results of the Harvard medical practice study I. New England Journal of Medicine, 324, 370-376.

[2] Bates, D.W., Cohen, M.S., Leape, L.L, Overhage, J.M., Sheridan, T. (2001). Reducing the frequency of errors in medicine using information technology. Journal of the American Medical Association, 8(4), 299-307.

[3] Bates, D.W., Teich, J.M., \& Shea, B. (1998). Effect of computerized physician order entry and a team intervention on prevention of serious medication errors. Journal of the American Medical Association, 280(15), 1311-1316.

[4] Balas, A.E. (2001). Information systems can prevent errors and improve quality. Journal of the American Medical Association, 8(4), 398-399.

[5] Evans, R.S., Pestotnik, M.S., Classen, D.C., Clemmer, T.P., Weaver, L.K., Orme, J.F. et al. (1998). A computer-assisted management program for antibiotics and other antiinfective agents. New England Journal of Medicine, 338(4), 232-238.

[6] Rind, D.M., Safran, C., Phillips, R.S., Wang, Q., Calkins, D.R., Delbanco, T.L. et al. (1994). Effect of computer-based alerts on the treatment and outcomes of hospitalized patients. Archives of Internal Medicine, 154, 1511-1517.

[7] Arkes, H.R., Shaffer, V.A., \& Medow, M.A. (2007). Patients derogate physicians who use a computer-assisted diagnostic aid. Medical Decision Making, 27, 1-14. 